\title{
Molecular Imaging of Cardiac Amyloidosis
}

\author{
Ahmad Masri ${ }^{1}$, Syed Bukhari², Yvonne S. Eisele ${ }^{2}$, and Prem Soman ${ }^{2}$ \\ ${ }^{1}$ Division of Cardiology, Oregon Health and Science University, Portland, Oregon; and ${ }^{2}$ Division of Cardiology, University of \\ Pittsburgh, Pittsburgh, Pennsylvania
}

Transthyretin and light-chain amyloidosis are the 2 main causes of cardiac amyloidosis. Recent developments in molecular imaging have transformed our ability to diagnose transthyretin cardiac amyloidosis noninvasively and unmasked a hitherto unrecognized prevalence of the disease. This review summarizes the current and evolving imaging approaches, their molecular structural basis, and the gaps in imaging capabilities that have arisen as a result of parallel developments in pharmacotherapy delivering the first effective treatment options for this condition.

Key Words: molecular imaging; SPECT; ATTR; bone-seeking tracers; cardiac amyloidosis

J Nucl Med 2020; 61:965-970

DOI: 10.2967/jnumed.120.245381

\footnotetext{
C
} ardiac amyloidosis is considered to be a rare disease, defined in the United States as a condition that affects fewer than 200,000 people (1). However, the recent adoption of noninvasive diagnostic approaches has unmasked a hitherto unrecognized community prevalence of wild-type amyloid transthyretin (ATTR) cardiac amyloidosis (CA), reversing prior impressions of its lower prevalence than amyloid lightchain (AL) amyloidosis (40.5 cases per million) (2). Quite serendipitously, parallel developments in pharmacotherapy have resulted in the recent approval by the U.S. Food and Drug Administration of 3 new agents for treating ATTR amyloidosis, with many clinical studies under way of new agents and expanded applications of approved ones. Taken together, these developments in diagnosis and therapy have transformed and invigorated the field of CA, which, until quite recently, relied solely on an invasive myocardial biopsy for diagnosis and faced therapeutic nihilism for ATTR amyloidosis. These developments have also spurred a renewed interest in the imaging of $\mathrm{CA}$, with emphasis on diagnosis, differentiation of amyloid type, and monitoring of therapy.

\footnotetext{
Received Mar. 27, 2020; revision accepted May 18, 2020.

For correspondence or reprints contact: Prem Soman, University of Pittsburgh Medical Center, A-429 Scaife Hall, 200 Lothrop St., Pittsburgh, PA 15213.

E-mail: premsoman@usa.net

Published online Jun. 1, 2020.

COPYRIGHT (c) 2020 by the Society of Nuclear Medicine and Molecular Imaging.
}

\section{THE MOLECULAR BASIS OF AMYLOIDOSIS}

The term amyloid was coined by Schleiden and used by Virchow in the mid-19th century based on the property of staining with iodine (amyloid = starchlike) (3). However, today we know that amyloid is derived from the misfolding of proteins (not starch) into long, unbranched fibrils of approximately $10 \mathrm{~nm}$ in diameter and several micrometers in length with a specific $\beta$-sheet structure that is extremely stable and resistant to degradation by cells. Amyloid diseases are generally chronic and occur because of progressive extracellular accumulation of amyloid in tissues, resulting in impaired function of the affected organs.

More than 30 different specific amyloid diseases have been described, with organ tropism and clinical manifestations driven by the protein of origin. Examples include Alzheimer disease, $\beta-2$ microglobulin-related amyloidosis in chronic dialysis patients, and systemic amyloid A amyloidosis associated with chronic inflammation. Irrespective of the protein of origin, all amyloid is morphologically indistinguishable and characterized by an antiparallel $\beta$-sheet secondary structure (in which the $\mathrm{N}$ - and $\mathrm{C}$ - terminals are oriented in opposite directions), which gives it its characteristic imaging properties with Congo red (red deposits on light microscopy and apple-green birefringence under polarized-light microscopy) and thioflavin T (yellow-green fluorescence) (2). The heterogeneity of organ involvement and clinical syndromes, despite morphologic homogeneity, is one of the mysteries of this intriguing disease.

The majority (95\%) of clinical CA is due to deposition of either transthyretin (TTR) or immunoglobulin AL-derived amyloid, which produce clinical diseases that differ vastly in their phenotypic expression, prognosis, and therapy. Rare cases of CA are derived from other precursor proteins, such as apolipoprotein A1. Amyloid fibril type is most specifically confirmed with immunohistochemistry or mass spectroscopy of biopsy material.

$\mathrm{AL}$ amyloidosis is the result of the unregulated proliferation of a single clone of plasma cells (a monoclonal gammopathy) resulting in the overproduction of immunoglobulin ALs that transform into amyloid in tissues. Certain isotypes $(\lambda)$ and variability subgroups $\left(\lambda_{\mathrm{VI}}\right)$ seem to confer a tendency to amyloidogenesis, since only a small proportion of patients with a plasma cell dyscrasia actually develop clinical amyloidosis (4). AL is a systemic disease with prognosis driven 
largely by the toxic-infiltrative cardiomyopathy (5). Hence, early diagnosis before advanced cardiac involvement that precludes chemotherapy is a key factor determining survival, potentially giving diagnostic imaging a key role in the evaluation of these patients. One of the critical steps in the evaluation (including imaging) of the patient with suspected $\mathrm{CA}$ is the identification of the amyloid type, since a mistaken diagnosis of ATTR for AL amyloidosis can have disastrous consequences.

ATTR amyloidosis is derived from TTR, a carrier of thyroxine and retinol bound to retinol-binding protein that is produced largely by the liver and, in smaller amounts, by the retinal epithelium and choroid plexus. The transformation of this 127-amino-acid protein into amyloid is stimulated by either unknown mechanisms related to aging in wild-type ATTR or by at least 120 known point mutations in its gene located on the long arm of chromosome 18, resulting in single-amino-acid substitutions in variant ATTR. Specific mutations result in well-described but not exclusive cardiac, neuropathic, or mixed phenotypes. The most common TTR gene mutation worldwide is V30M. In the United States, the V122I mutation is the most prevalent and is carried by $3 \%-$ $4 \%$ of the African American population (6). In contrast to AL amyloidosis, the cardiac consequences of ATTR appear to result mostly from infiltration of the myocardium, with less evidence of direct toxic effects.

Contemporary clinical data suggest that there is generally a lag period of 2 or more years between the patient's first presentation to a physician and the diagnosis of CA (7). Recent educational efforts have focused on increasing awareness of red-flag clinical features. In this context, imaging plays a key role in the early and accurate diagnosis of CA.

\section{ESTABLISHED IMAGING APPROACHES}

Contemporary imaging approaches are listed in Table 1 and illustrated in Figure 1. On echocardiography, the initial suspicion of CA is often spurred by the nonspecific finding of increased left ventricular thickness, which can be asymmetric in up to $20 \%$ of patients (8). Other findings on echocardiography include left and right atrial enlargement, increased thickness of the right ventricular walls and the interatrial septum, pericardial effusion, diastolic dysfunction, and a longitudinal strain abnormality with relative apical sparing (7). A previously described granular speckled appearance of the myocardium is now thought to be nonspecific for amyloidosis. Diagnostic confirmation of amyloidosis generally requires a tissue biopsy, and echocardiographic findings do not distinguish between ATTR and AL CA. Given the nonspecific nature of echocardiography findings, its diagnostic accuracy even in combination with electrocardiography findings is only $60 \%(9)$.

\section{Cardiac MR}

In addition to the morphologic abnormalities seen on echocardiography, a unique abnormality of gadolinium kinetics, seen as an inability to null the myocardium on the inversion time mapping sequence along with early clearance of tracer from the blood pool, is typical. Native T1 time and the extracellular volume fraction are generally higher in amyloidosis than other commonly encountered conditions producing increased left ventricular thickness, and a high extracellular volume is associated with a particularly negative prognosis in amyloidosis (10). Cardiac MR features do not reliably distinguish between ATTR and AL CA, and the possibility of a negative cardiac MR result in biopsy-proven amyloidosis is reported to be up to $12 \%(9)$.

TABLE 1

Radionuclide Tracers for Imaging of Amyloidosis

\begin{tabular}{|c|c|c|}
\hline Tracer & Molecular target & Disease target \\
\hline \multicolumn{3}{|l|}{ SPECT tracers } \\
\hline Bone-seeking radiotracers & Phosphate binders & ATTR \\
\hline \multicolumn{3}{|l|}{ 99mTc-pyrophosphate } \\
\hline \multicolumn{3}{|c|}{ 99mTc-3,3-diphosphono-1,2-propanodicarboxylic acid } \\
\hline \multicolumn{3}{|c|}{ 99mTc-hydroxymethylene diphosphonate } \\
\hline \multicolumn{3}{|c|}{ Other radiotracers } \\
\hline${ }^{123} \mathrm{I}$-serum amyloid protein & Serum amyloid $P$ & All amyloidoses \\
\hline 123/-aprotinin & Serum protease inhibitor, amyloid binder & All amyloidoses \\
\hline \multicolumn{3}{|l|}{ PET tracers } \\
\hline Thioflavin-T derivatives & All amyloid fibrils ( $\beta$-structure and side chains) & $\mathrm{AL}$ and $\mathrm{ATTR}$ \\
\hline \multicolumn{3}{|l|}{${ }^{18} \mathrm{~F}$-florbetapir } \\
\hline \multicolumn{3}{|l|}{ 18F-florbetaben } \\
\hline \multicolumn{3}{|l|}{${ }^{18} \mathrm{~F}$-flutemetamol } \\
\hline \multicolumn{3}{|l|}{${ }^{11} \mathrm{C}-$ Pittsburgh compound B } \\
\hline${ }^{18} \mathrm{~F}$-sodium fluoride & Microcalcification & ATTR \\
\hline${ }^{124} \mathrm{I}-\mathrm{m} 11-1 \mathrm{~F} 4$ monoclonal antibody & Immunoglobulin ALs & $\mathrm{AL}$ \\
\hline
\end{tabular}




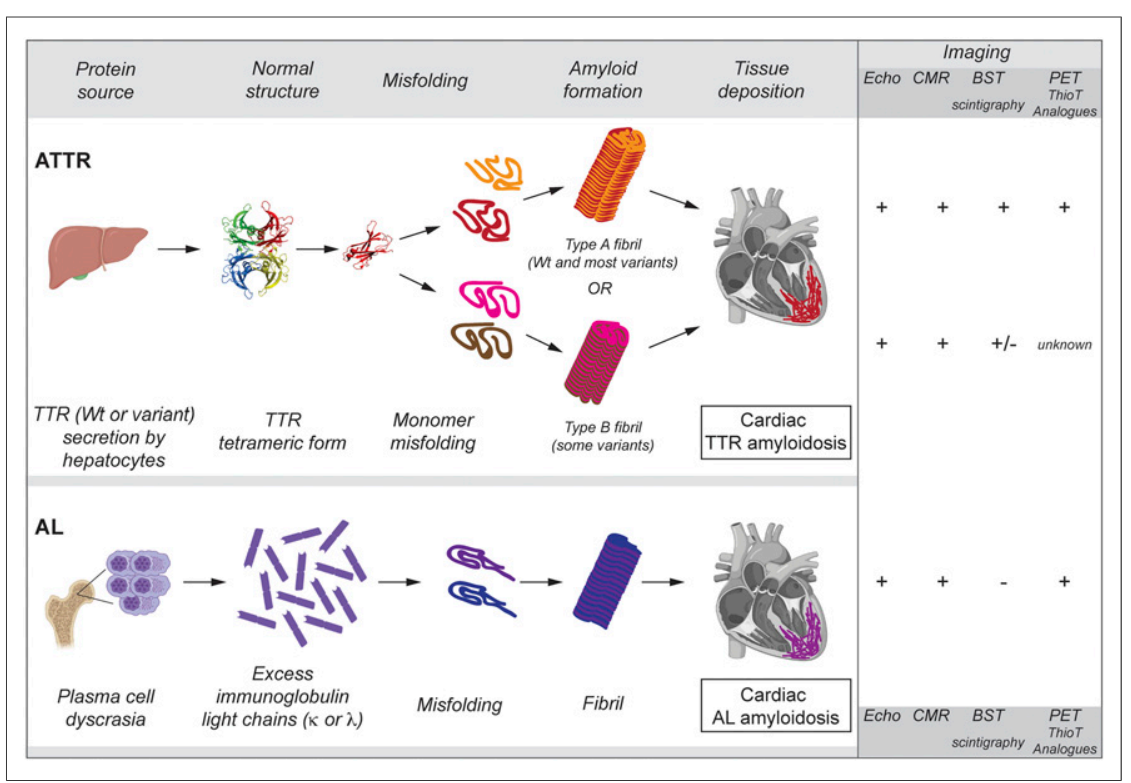

FIGURE 1. Molecular mechanism of amyloidosis and imaging characteristics illustrated for ATTR amyloidosis and AL amyloidosis. Echocardiography, CMR, and thioflavin-analog PET tracers detect both types, whereas $99 \mathrm{mTc}$-labeled bone tracers (BST) are avidly taken up only by ATTR amyloidosis. Notably, hereditary amyloidosis resulting from some mutations result in type B amyloid fibrils, which are less avid for BST. On the other hand, prior reports suggest that some patients with AL may show BST uptake. Differentiation of ATTR from AL amyloidosis is a critical step in evaluation of patients with suspected CA. Wt = wild-type.

\section{Radionuclide Scintigraphy with Bone-Seeking Tracers}

A resurgence of interest and an improved understanding of the performance characteristics of myocardial scintigraphy with the ${ }^{99 \mathrm{~m}}$ Tc-labeled bone-seeking tracers ${ }^{99 \mathrm{~m}} \mathrm{Tc}$-pyrophosphate, 3,3-diphosphono-1,2-propanodicarboxylic acid ( ${ }^{99 \mathrm{~m} T c-D P D),}$ and ${ }^{99 m}$ Tc-hydroxymethylene diphosphonate have been transformative for the field of CA. A recent multinational study of 1,212 patients reported a high sensitivity and near-perfect specificity for ATTR CA if plasma cell disease is excluded by serum studies (11), and a metaanalysis of 5 studies found a sensitivity of $92.2 \%$ (95\% confidence interval, 89\%-95\%) and specificity of $95.4 \%(97 \%-99 \%)$ (12). This tropism of the bone-seeking tracers for ATTR, but not AL, amyloidosis is probably attributable to calcium binding (13). Cardiac scintigraphy has greatly expanded the pool of patients being tested for CA and obviated a myocardial biopsy in cases of clinical suspicion with positive scintigraphy and negative serum studies. Reports suggest an unexpectedly high prevalence of ATTR amyloidosis in defined populations such as patients with heart failure with preserved ejection fraction and even mildly increased left ventricular wall thickness (13\%), patients with paradoxic low-gradient severe aortic stenosis (10\%), and African American patients with heart failure with preserved ejection fraction in the United States (6).

Among the bone-seeking tracers useful in CA, ${ }^{99} \mathrm{~m}$ Tcpyrophosphate is available in the United States. Of note, ${ }^{99 m}$ Tc-methyldiphosphonate, which is widely used for bone scans, is not useful for amyloidosis imaging because of poor sensitivity. ${ }^{99 m}$ Tc-pyrophosphate scintigraphy is a relatively simple test that is widely available and applicable in a broad range of patients, including those with relative contraindications to other imaging modalities, such as atrial fibrillation, implanted cardiac devices, contrast allergy, renal dysfunction, and extreme obesity. The combination of high clinical efficacy (diagnostic accuracy) and clinical effectiveness (applicability) make it highly useful for screening.

Interpretation is based on a semiquantitative visual grading of tracer uptake, first proposed by Perugini (grade $0=$ no myocardial uptake, grade $1=$ myocardial $<$ rib uptake, grade $2=$ myocardial equal to ribs, and grade $3=$ myocardial $>$ rib uptake, usually with diminished bone uptake) (14). Grades $\geq 2$ are considered positive. Figure 2 shows a typical example of a positive ${ }^{99 \mathrm{~m} T c-}$ pyrophosphate scan. Prior reports of ${ }^{99 m}$ Tc-pyrophosphate uptake in some patients with AL amyloidosis have prompted a strong recommendation for mandatory serum studies (serum-free AL assay and immunofixation electrophoresis) in all patients undergoing scintigraphy (15). A quantitative heart-to-contralateral ratio has been proposed for ${ }^{99 \mathrm{~m}} \mathrm{Tc}$-pyrophosphate, with values greater than 1.5 being highly specific for ATTR (16). Guidelines recommend imaging at 1 or $3 \mathrm{~h}$ (or both) after tracer injection. The principal confounder of planar imaging is the presence of tracer in the cardiac blood pool resulting in a spuriously elevated planar grade and a false-positive result. SPECT imaging is extremely useful to distinguish between blood pool and myocardial tracer (Fig. 3). More recent prospective studies indicate no added advantage of 3-h imaging when both planar and SPECT imaging are performed at $1 \mathrm{~h}$ (17). A major disadvantage of 99mTc-pyrophosphate scintigraphy is the lack of practical approaches for quantification of absolute tracer uptake and serial imaging of disease burden, an important need in the current era of amyloid pharmacotherapy. Occasionally, an incidental finding of myocardial uptake is noted in ${ }^{99 \mathrm{~m}}$ Tc-labeled bone scans. We feel that the referring physician should be alerted to this finding, but its prognostic and therapeutic implications in the asymptomatic patient remain unclear.

\section{EVOLVING IMAGING APPROACHES}

Several PET tracers have been studied for CA, including the thioflavin $\mathrm{T}$ analogs ${ }^{11} \mathrm{C}$-Pittsburgh compound $\mathrm{B}$ (18), 


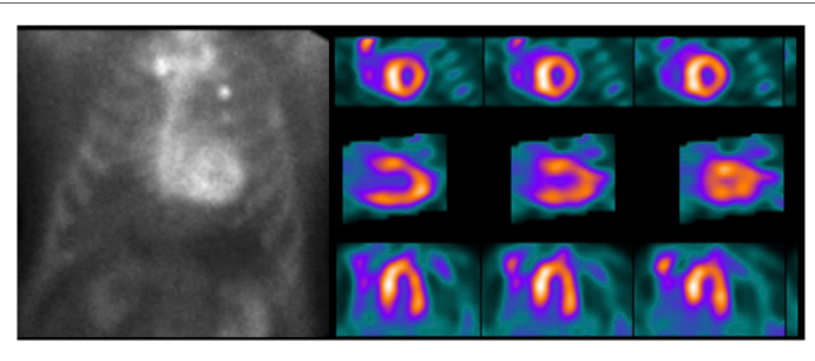

FIGURE 2. (Left) 99mTc-pyrophosphate planar scan showing avid myocardial tracer uptake (Perugini grade 3, myocardial uptake greater than rib). (Right) Cardiac SPECT showing diffuse myocardial tracer uptake in short-axis images (top row), verticallong axis images (middle row), and horizontal long-axis (bottom row). This scan is diagnostic of ATTR cardiomyopathy if serum and urine studies for AL amyloidosis are negative.

${ }^{18} \mathrm{~F}$-florbetapir (19), ${ }^{18} \mathrm{~F}$-florbetaben $(20)$, and ${ }^{18} \mathrm{~F}$-flutemetamol (21). These agents were all developed for imaging cerebral $\mathrm{A} \beta$-amyloid in Alzheimer disease and are taken up by myocardial amyloid. They do not, however, distinguish between ATTR and AL. A major impetus for the development of amyloid PET tracers has been the expectation of more accurate quantification abilities than are possible with SPECT. In preliminary studies, quantitative metrics show higher myocardial uptake in patients with AL or ATTR CA than in controls $(19,22)$, in AL patients than in ATTR patients (23), and in chemotherapy-naïve patients than in postchemotherapy patients $(24) .{ }^{18} \mathrm{~F}-\mathrm{NaF}$, an agent used to image bone metastases in several types of cancer, targets microcalcifications. Small studies suggest utility for the diagnosis of ATTR CA, with quantitative parameters able to distinguish patients with ATTR CA from AL CA and controls (25). The affinity of ${ }^{18} \mathrm{~F}-\mathrm{NaF}$ for ATTR CA corroborates the calcium hypothesis to explain the binding of bone-seeking tracers to ATTR. Serum amyloid P component is a circulating plasma protein that has high affinity for amyloid fibrils and is a nonfibrillar component of all types of amyloid. Theoretically, SAP targeted imaging should be specific for tissue amyloid. However, the SPECT tracer ${ }^{123}$ ISAP was found to be less useful for cardiac imaging because of high blood-pool activity and poor count statistics in the myocardium (26). Another SPECT tracer, ${ }^{99 \mathrm{~m} T c-a p r o t i n i n, ~ t a r-~}$ gets this serum protease inhibitor found in amyloid deposits. However, its utility is also limited by poor-quality images (27). Similarly, a murine monoclonal antibody developed against ALs and labeled with iodine was found to have limited use in CA (28)

\section{BINDING MECHANISMS: INSIGHTS FROM STRUCTURE-FUNCTION RELATIONSHIPS}

The structure of TTR-derived amyloid fibrils was recently revealed using cryoelectron microscopy (29). Perturbations in the structural conformation of the precursor protein are at the center of amyloidogenesis. Interestingly, at least 2 identified rescue mutations, T119M and R104H, stabilize TTR in patients who are compound heterozygotes for a pathogenic mutation and prevent expression of disease (30). Despite the heterogeneity of the mechanisms leading to amyloid formation (aging, gene mutation, proteolytic remodeling), the amyloid fibril is characterized by relative structural homogeneity. Early studies exploring the binding mechanism of thioflavin T suggested that the surface of the cross $\beta$ structures forms the binding site, and the orientation of the side chains may be the common pattern that determines the affinity of thioflavin $\mathrm{T}$ to a broad range of amyloid fibrils (31). The binding mechanism of thioflavin $\mathrm{T}$ is shared by analogous tracers, including ${ }^{11} \mathrm{C}$-Pittsburgh compound $\mathrm{B}$ and the ${ }^{18} \mathrm{~F}$-tracers (Fig. 4). The SPECT and PET tracers bind by different, not yet fully elucidated, mechanisms. It would seem logical to attribute the tropism of the

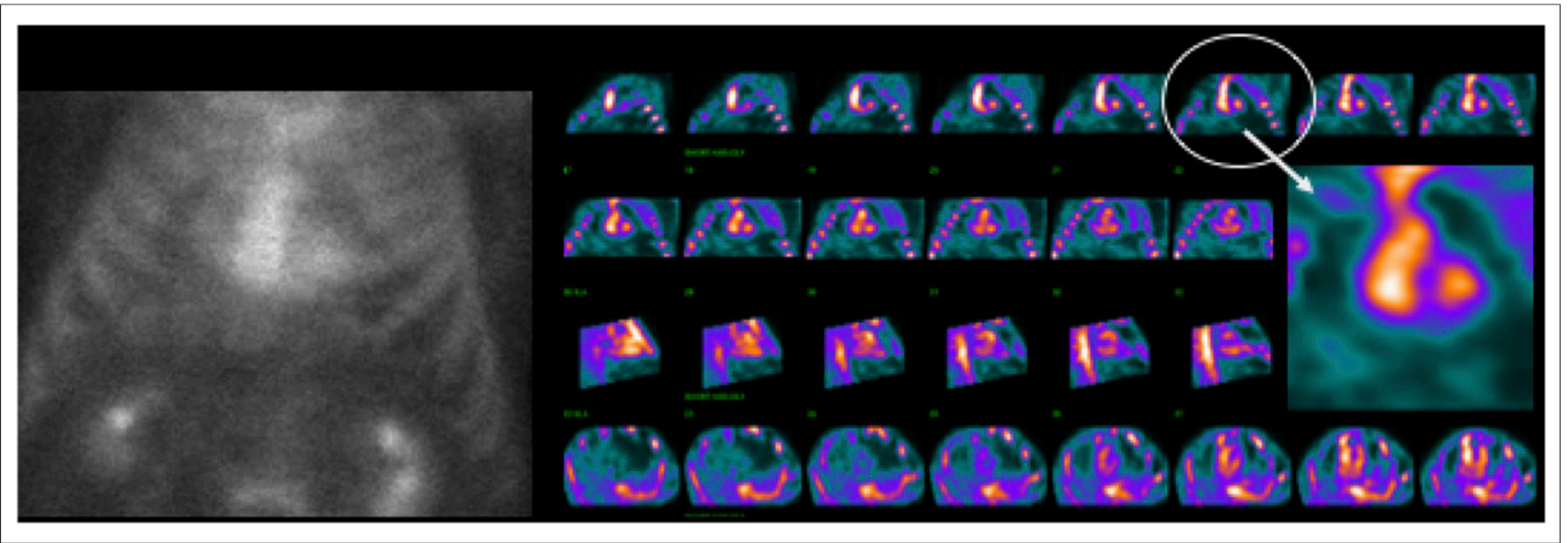

FIGURE 3. (Left) ${ }^{99 m T c-p y r o p h o s p h a t e ~ p l a n a r ~ s c a n ~ s h o w i n g ~ m i l d ~ m y o c a r d i a l ~ t r a c e r ~ u p t a k e ~(P e r u g i n i ~ g r a d e ~ 1, ~ m y o c a r d i a l ~ u p t a k e ~}$ of tracer less than rib). (Right) Chest SPECT images showing tracer in left ventricular cavity as seen best in coronal views (top 2 rows and magnified image) compared with sagittal view (third row from top) or transaxial view (bottom row), illustrating value of SPECT for differentiating blood pool from myocardial activity. In cases without myocardial tracer uptake, it is easier to perform chest reconstruction than cardiac reconstruction. 


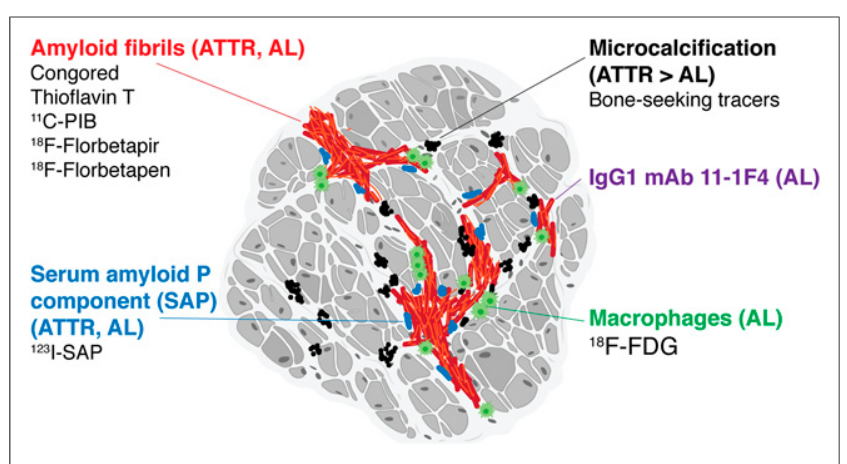

FIGURE 4. Congo red, thioflavin $\mathrm{T}$, and its analogs ${ }^{11} \mathrm{C}-\mathrm{PIB}$, ${ }^{18} \mathrm{~F}$-florbetapir, and ${ }^{18} \mathrm{~F}$-florbetapen bind to both ATTR and AL amyloid fibrils. $99 \mathrm{mTc}$ bone-seeking tracers show avid uptake in ATTR, possibly related to microcalcification. Increased uptake of ${ }^{18} \mathrm{~F}-\mathrm{FDG}$ in $\mathrm{AL}$ is attributed to inflammation and macrophage infiltration. Serum amyloid $P$ component (SAP) in ${ }^{123}$-SAP binds to both ATTR and AL fibrils. 11-1F4 is monoclonal antibody against immunoglobulin ALs.

SPECT bone-seeking tracers to calcium binding; however, there are only sparse clinical data to support this notion. To specifically explore tracer binding of the bone-seeking tracers, Stats et al. performed a unique study of endomyocardial biopsies from 8 ATTR and AL amyloidosis patients (13). Using specific stains and immunohistochemistry, they reported a greater density of microcalcification in biopsies from ATTR patients and a greater density of macrophages in biopsies from AL patients, supporting a calcium-based binding mechanism of bone tracer uptake in ATTR. In this regard, it is relevant that ${ }^{99 \mathrm{~m}} \mathrm{Tc}$-pyrophosphate was introduced as a tracer for the imaging of subacute myocardial infarction, where its uptake correlates with calcium deposits in the infarcted myocardium (32), and that ${ }^{18} \mathrm{~F}-\mathrm{NaF}$ is a tracer that specifically binds to sites of active microcalcification. These facts further support a calcium hypothesis to explain the binding of these tracers. Of clinical relevance, some of the cases of AL in the study of Stats et al. demonstrated densities of microcalcification comparable to ATTR cases, consistent with the clinical finding of ${ }^{99 \mathrm{~m}} \mathrm{Tc}-$ $\mathrm{DPD} / 9{ }^{9 m} \mathrm{Tc}-$ pyrophosphate positivity in some patients with $\mathrm{AL}$ amyloidosis.

The mechanistic basis of increased calcification in ATTR is unknown. The density of calcification in the study of Stats et al. was unrelated to age, cardiac function, renal function, or serum calcium. The authors postulate that the denser microcalcification in ATTR may be a reflection of chronicity compared with the typical time course of AL. The finding of a higher density of macrophages in biopsies from patients with localized AL is consistent with prior studies showing ${ }^{18} \mathrm{~F}-\mathrm{FDG}$ uptake in these patients (33).

Although amyloid fibrils derived from different precursor proteins are mostly structurally homogeneous, recent reports using Western blot analysis have indicated the existence of 2 types of amyloid fibrils derived from TTR. Type A fibrils consist of a mixture of C-terminal fragments (N-terminally truncated) and full-length TTR and are the predominant type in wild-type ATTR and most hATTR. Type B fibrils consist only of full-length TTR and are found in some patients with V30M ATTR and have been reported with the T114C mutation (34). The fibril type in V30M determines the clinical phenotype. Patients with type A are characterized by late-onset (age $>50 \mathrm{y}$ ) disease with a mixture of neuropathy and cardiomyopathy. Patients with type B have early-onset disease that has a predominantly neuropathic phenotype. Geographic clustering occurs, with early-onset (third decade) disease prevalent in Portugal, Japan, and Brazil, and a mixture of early- and late-onset disease (fifth to sixth decades) common in Sweden (35). Individual patients with hATTR display only one type of fibril, which does not change over time (36). Type A fibrils are less avid for Congo red than type B fibrils, a fact that may explain the lower yield of subcutaneous-fat-pad biopsies in wild-type ATTR (35).

Pilebro et al. reported that patients with type B fibrils were ${ }^{99 \mathrm{~m}}$ Tc-DPD-negative, despite echocardiographic and, in some patients, cardiac MR evidence of cardiac involvement (37). A low sensitivity for ${ }^{99 \mathrm{~m}} \mathrm{Tc}-\mathrm{DPD}$ has also been reported for ATTR because of the P64L mutation (38). Thus, calcification alone cannot fully explain the affinity of bone-seeking tracers to ATTR, and direct binding to fibrils cannot be excluded at this time. However, these small studies did not confirm cardiac involvement by biopsy and, therefore, are far from conclusive.

\section{KNOWLEDGE GAPS AND FUTURE DIRECTIONS}

With the advent of effective treatment options for ATTR amyloidosis, a critical need has emerged for an imaging test to monitor disease activity by serial imaging. All current imaging approaches have limitations in this regard, including scintigraphy with bone-seeking tracers and cardiac MR $(39,40)$. With its superior ability to quantify tracer uptake, PET-based tracers are being actively investigated for serial imaging. An improved understanding of structure-function relationships and binding mechanism will help in efforts to develop new amyloid tracers.

Despite the transformative effects of scintigraphy on our ability to diagnose ATTR CA, the global experience with these tracers for the diagnosis of CA is still relatively small. Its performance characteristics in the community setting (in contrast to specialist centers with highly selected referral populations) remain unexplored. Conditions other than CA resulting in positive scintigraphy are being identified (e.g., hydroxychloroquine cardiotoxicity) (41). Binding mechanisms and the effect of fibril type on tracer affinity need to be clarified. Thus, whereas scintigraphy with the ${ }^{99 \mathrm{~m}} \mathrm{Tc}$ bone-seeking tracers is a remarkably useful test for patients with suspected amyloidosis, vigilance is required to identify and further evaluate cases in which there is discordance between clinical and imaging findings.

\section{DISCLOSURE}

Ahmad Masri received research funding from Pfizer and Akcea. Prem Soman received grant funding from Pfizer and 
is on the advisory board of Pfizer, Alnylam, and Eidos. Syed Bukhari is the current incumbent of a CA fellowship funded by Pfizer through a competitive national RFA. Yvonne Eisele and Prem Soman received support from the Vascular Medicine Institute, University of Pittsburgh. Yvonne Eisele received support from the National Institute on Aging under award number R00 AG050764. No other potential conflict of interest relevant to this article was reported.

\section{ACKNOWLEDGMENT}

Figures 1 and 4 were partly created with BioRender.com.

\section{REFERENCES}

1. Orphan drug regulations: regulatory history. U.S. Food and Drug Administration website. http://www.fda.gov/ForIndustry/DevelopingProductsforRareDiseasesConditions/ HowtoapplyforOrphanProductDesignation/ucm356481.htm. Revised February 28, 2018. Accessed June 5, 2020.

2. Quock TP, Yan T, Chang E, Guthrie S, Broder MS. Epidemiology of AL amyloidosis: a real-world study using US claims data. Blood Adv. 2018;2:1046-1053.

3. Maleszewski JJ. Cardiac amyloidosis: pathology, nomenclature, and typing. Cardiovasc Pathol. 2015;24:343-350.

4. Merlini G, Bellotti V. Molecular mechanisms of amyloidosis. $N$ Engl J Med. 2003;349:583-596

5. Shi J, Guan J, Jiang B, et al. Amyloidogenic light chains induce cardiomyocyte contractile dysfunction and apoptosis via a non-canonical p38 $\alpha$ MAPK pathway. Proc Natl Acad Sci USA. 2010;107:4188-4193.

6. Ruberg FL, Grogan M, Hanna M, Kelly JW, Maurer MS. Transthyretin amyloid cardiomyopathy: JACC state-of-the-art review. J Am Coll Cardiol. 2019;73:2872-2891.

7. Maurer MS, Elliott P, Comenzo R, Semigran M, Rapezzi C. Addressing common questions encountered in the diagnosis and management of cardiac amyloidosis. Circulation. 2017;135:1357-1377.

8. González-López E, Gagliardi C, Dominguez F, et al. Clinical characteristics of wild-type transthyretin cardiac amyloidosis: disproving myths. Eur Heart J. 2017;38:1895-1904.

9. Austin BA, Tang WH, Rodriguez ER, et al. Delayed hyper-enhancement magnetic resonance imaging provides incremental diagnostic and prognostic utility in suspected cardiac amyloidosis. JACC Cardiovasc Imaging. 2009;2:1369-1377.

10. Wong TC, Piehler KM, Zareba KM, et al. Myocardial damage detected by late gadolinium enhancement cardiovascular magnetic resonance is associated with subsequent hospitalization for heart failure. J Am Heart Assoc. 2013;2:e000416.

11. Gillmore JD, Maurer MS, Falk RH, et al. Nonbiopsy diagnosis of cardiac transthyretin amyloidosis. Circulation. 2016;133:2404-2412.

12. Treglia G, Glaudemans A, Bertagna F, et al. Diagnostic accuracy of bone scintigraphy in the assessment of cardiac transthyretin-related amyloidosis: a bivariate meta-analysis. Eur J Nucl Med Mol Imaging. 2018;45:1945-1955.

13. Stats MA, Stone JR. Varying levels of small microcalcifications and macrophages in ATTR and AL cardiac amyloidosis: implications for utilizing nuclear medicine studies to subtype amyloidosis. Cardiovasc Pathol. 2016;25:413-417.

14. Perugini E, Guidalotti PL, Salvi F, et al. Noninvasive etiologic diagnosis of cardiac amyloidosis using 99m Tc-3,3-diphosphono-1,2-propanodicarboxylic acid scintigraphy. J Am Coll Cardiol. 2005;46:1076-1084.

15. Dorbala S, Ando Y, Bokhari S, et al. ASNC/AHA/ASE/EANM/HFSA/ISA/ SCMR/SNMMI expert consensus recommendations for multimodality imaging in cardiac amyloidosis: part 1 of 2-evidence base and standardized methods of imaging. J Nucl Cardiol. 2019;26:2065-2123.

16. Bokhari S, Castaño A, Pozniakoff T, Deslisle S, Latif F, Maurer MS. ${ }^{99 m T c-}$ pyrophosphate scintigraphy for differentiating light-chain cardiac amyloidosis from the transthyretin-related familial and senile cardiac amyloidoses. Circ Cardiovasc Imaging. 2013;6:195-201.

17. Masri A, Bukhari S, Ahmad S, et al. Efficient 1-hour technetium-99 m pyrophosphate imaging protocol for the diagnosis of transthyretin cardiac amyloidosis. Circ Cardiovasc Imaging. 2020;13:e010249.
18. Mathis CA, Wang Y, Holt DP, Huang GF, Debnath ML, Klunk WE. Synthesis and evaluation of ${ }^{11} \mathrm{C}$-labeled 6-substituted 2-arylbenzothiazoles as amyloid imaging agents. J Med Chem. 2003;46:2740-2754.

19. Dorbala S, Vangala D, Semer J, et al. Imaging cardiac amyloidosis: a pilot study using ${ }^{18} \mathrm{~F}$-florbetapir positron emission tomography. Eur J Nucl Med Mol Imaging. 2014;41:1652-1662.

20. Law WP, Wang WYS, Moore PT, Mollee PN, Ng ACT. Cardiac amyloid imaging with ${ }^{18}$ F-florbetaben PET: a pilot study. J Nucl Med. 2016;57:1733-1739.

21. Kircher M, Ihne S, Brumberg J, et al. Detection of cardiac amyloidosis with ${ }^{18} \mathrm{~F}-$ Florbetaben-PET/CT in comparison to echocardiography, cardiac MRI and DPDscintigraphy. Eur J Nucl Med Mol Imaging. 2019;46:1407-1416.

22. Antoni $G$, Lubberink M, Estrada S, et al. In vivo visualization of amyloid deposits in the heart with ${ }^{11} \mathrm{C}$-PIB and PET. J Nucl Med. 2013;54:213-220.

23. Lee SP, Lee ES, Choi $\mathrm{H}$, et al. ${ }^{11} \mathrm{C}$-Pittsburgh B PET imaging in cardiac amyloidosis. JACC Cardiovasc Imaging. 2015;8:50-59.

24. Park MA, Padera RF, Belanger A, et al. ${ }^{18} \mathrm{~F}$-florbetapir binds specifically to myocardial light chain and transthyretin amyloid deposits: autoradiography study. Circ Cardiovasc Imaging. 2015;8: 10.1161/CIRCIMAGING.114.002954.

25. Morgenstern R, Yeh R, Castano A, Maurer MS, Bokhari S. ${ }^{18}$ Fluorine sodium fluoride positron emission tomography, a potential biomarker of transthyretin cardiac amyloidosis. J Nucl Cardiol. 2018;25:1559-1567.

26. Hazenberg BP, van Rijswijk MH, Lub-de Hooge MN, et al. Diagnostic performance and prognostic value of extravascular retention of ${ }^{123} \mathrm{I}$-labeled serum amyloid $\mathrm{P}$ component in systemic amyloidosis. J Nucl Med. 2007;48:865-872.

27. Minamimoto R, Kubota K, Ishii K, et al. Re-evaluating the potentials and limitations of ${ }^{99 \mathrm{~m} T c-a p r o t i n i n}$ scintigraphy for amyloid imaging. Am J Nucl Med Mol Imaging. 2013;3:261-271.

28. Wall JS, Kennel SJ, Stuckey AC, et al. Radioimmunodetection of amyloid deposits in patients with AL amyloidosis. Blood. 2010;116:2241-2244.

29. Schmidt M, Wiese S, Adak V, et al. Cryo-EM structure of a transthyretin-derived amyloid fibril from a patient with hereditary ATTR amyloidosis. Nat Commun. 2019;10:5008

30. Hornstrup LS, Frikke-Schmidt R, Nordestgaard BG, Tybjaerg-Hansen A. Genetic stabilization of transthyretin, cerebrovascular disease, and life expectancy. Arter Thromb Vasc Biol. 2013;33:1441-1447.

31. Biancalana M, Koide S. Molecular mechanism of thioflavin-T binding to amyloid fibrils. Biochim Biophys Acta. 2010;1804:1405-1412.

32. Reimer KA, Martonffy K, Schumacher BL, Henkin RE, Quinn JL III, Jennings RB. Localization of ${ }^{99 \mathrm{~m} T c}$-labeled pyrophosphate and calcium in mycoardial infarcts after temporary coronary occlusion in dogs. Proc Soc Exp Biol Med. 1977; 156:272-276.

33. Glaudemans AWJM, Slart RHJA, Noordzij W, Dierckx RAJO, Hazenberg BPC. Utility of ${ }^{18} \mathrm{~F}-\mathrm{FDG}$ PET(/CT) in patients with systemic and localized amyloidosis. Eur J Nucl Med Mol Imaging. 2013;40:1095-1101.

34. Ihse E, Rapezzi C, Merlini G, et al. Amyloid fibrils containing fragmented ATTR may be the standard fibril composition in ATTR amyloidosis. Amyloid. 2013;20:142-150.

35. Suhr OB, Lundgren E, Westermark P. One mutation, two distinct disease variants: unravelling the impact of transthyretin amyloid fibril composition. $J$ Intern Med. 2017;281:337-347.

36. Ihse E, Suhr OB, Hellman U, Westermark P. Variation in amount of wild-type transthyretin in different fibril and tissue types in ATTR amyloidosis. J Mol Med. 2011;89:171-180.

37. Pilebro B, Suhr OB, Naslund U, Westermark P, Lindqvist P, Sundstrom T. ${ }^{99 \mathrm{~m}}$ TcDPD uptake reflects amyloid fibril composition in hereditary transthyretin amyloidosis. Ups J Med Sci. 2016;121:17-24.

38. Musumeci MB, Cappelli F, Russo D, et al. Low sensitivity of bone scintigraphy in detecting Phe64Leu mutation-related transthyretin cardiac amyloidosis. JACC Cardiovasc Imaging. 2020;13:1314-1321.

39. Castaño A, DeLuca A, Weinberg R, et al. Serial scanning with technetium pyrophosphate $\left({ }^{99 m}\right.$ Tc-PYP) in advanced ATTR cardiac amyloidosis. J Nucl Cardiol. 2016;23:1355-1363.

40. Ruberg FL, Fontana M, Gillmore JD. Should histologic determination of amyloid load determine management decisions in light-chain amyloidosis? J Am Coll Cardiol. 2016;68:2493-2494.

41. Layoun ME, Desmarais J, Heitner SB, Masri A. Hot hearts on bone scintigraphy are not all amyloidosis: hydroxychloroquine-induced restrictive cardiomyopathy. Eur Heart J. February 20, 2020. 